reflect and represent major adaptations to a particular way of life; that is the "adaptive plateau" discussed by Sewall Wright, Ernst Mayr and others. It is difficult, therefore, for some palaeoanthropologists to accept as generically distinctive the differences in certain features in Australopithecus africanus and Homo "habilis" which are probably due only to normal variation within a single species. Moreover, the slow evolution of a single, widespread population of Australopithecus into Homo would seem more consistent with modern evolutionary theory than with the continued existence of several generically and specifically distinct groups of hominids.

Criticisms of theory aside, it is interesting and surprising to find that the new Homo "habilis" from Olduvai does not fulfil all the generic criteria outlined in the 1964 revision. The revision calls for a minimum cranial capacity of "about $600 \mathrm{~cm}^{3}$ ". Although some gorillas have more than $600 \mathrm{~cm}^{8}$, Olduvai Hominid 24 has only $560 \mathrm{~cm}^{3}$. This is admittedly a preliminary estimate because there is "still some evidence of distortion in the reconstruction" and two inevitable questions arise: if cranial capacity cannot be accurately determined does this not undermine the validity of many of the cranial measurements? And does it not also undermine the validity of the conclusions that are drawn, at least in part, from those measurements?

One taxonomic criterion included in the 1964 revision was not phrased in equivocal terms: a member of the genus Homo will not have the concave or "dished" face characteristic of all known australopithecines. The dished face is a composite feature of considerable evolutionary significance and its absence in later hominines represents a diminution of the teeth and jaws, with reduced prognathism in the lower part of the face and emergence of the nose. The profile line drawing (Fig. 3 on page 310 ) clearly demonstrates the dished face of Olduvai Hominid 24 and serves to emphasize its overall similarity with the undoubted australopithecine from South Africa.

Olduvai Hominid 24 has been nicknamed "Twiggy" because its flattened shape before restoration resembled that notably unendowed figure. A badly crushed and distorted skull nearly two million years old presents some problems to those restoring it; it presents even more problems to those who try to decide, finally, what it is. On the basis of the evidence presented by Dr Leakey it is very difficult to agree that this specimen is "clearly Homo". Indeed it would seem to be excluded from that group on the basis of his own criteria are outlined in his 1964 article.
TRANSFORMATION Responses to Cyclic AMP

from our Cell Biology Correspondent EARLIER this year I drew attention in this column to the work of Puck and his associates and Pastan's group who respectively showed that dibutyryl adenosine cyclic $3^{\prime}, 5^{\prime}$ monophosphate causes Chinese hamster ovary cells growing in culture to assume reversibly a fibroblastic morphology, and causes the phenotypic reversion of transformed mouse fibroblasts. More recently Nature's Cell Physiology Correspondent (Nature New Biology, 232, 35; 1971) commented upon the experiments of Sheppard, who has shown that doses of this substituted cyclic AMP not only restore the growth control and susceptibility to contact inhibition of populations of 3T6 cells and 3T3 cells transformed by polyoma virus but also reduce the susceptibility of these cells to agglutination by wheat germ agglutinin.

\title{
Myoglobin Sequence Completed
}

THE torrent of articles concerned, on the one hand, with the structure and function of haemoglobins and on the other with the characterization of variant haemoglobins, shows no sign of abating. A welcome addition, however, is the appearance, in next Wednesday's Nature New Biology, of three articles on the sequence of human myoglobin, the choice of an allosteric model for the haemoglobin-oxygen system, and the relation between structure and properties of the Japanese human haemoglobin variant $\mathbf{H b}$-Hiroshima.

Herra and Lehmann have repeated some parts of Hill's sequence studies on human myoglobin and disagree with him on only three points. They have also completed the sequence which largely agrees with that proposed from considerations of amino-acid composition and homology with the known sequence of sperm whale myoglobin. Their sequence allows the differences between seven myoglobins to be delineated and will no doubt stimulate discussion on the homology and evolution of mammalian myoglobins. The unique feature of human myoglobin is a cysteine residue at position 110 (G11), which it has in common with the $\alpha$-chain of human haemoglobin. Apart from this feature, human myoglobin resembles the human $\alpha$-chain to no greater extent than the $\beta$-chain, having thirty-seven identical residues with each. of these several are discovered among the haem contacts of the $\alpha$ and $\beta$-chains.

The complete human myoglobin sequence will be of great value in determining the structural differences of the four known human skeletal myoglobin electrophoretic variants; the possible existence of neutral mutations can also be investigated. Herra and Lehmann report no differences in the fingerprints of human skeletal and heart myoglobin and the skeletal myoglobin of a case of Duchenne's muscular dystrophy. Further work on human myoglobin variants, which has in the past been an area of some confusion, will be awaited with interest.
Connoisseurs of theories of allosteric systems will no doubt appreciate a reexamination by Minton in next Wednesday's Nature New Biology of a recent treatment by Edelstein (Nature, 230,224 ; 1971) of the allosteric model for haemoglobin. Edelstein had concluded that the sequential model of Koshland, Nemethy and Filmer describes the oxygen binding curves of several species of human haemoglobin less well than does the two-state model of Monod, Wyman and Changeux. Minton points out that a tacit assumption in Edelstein's analysis is likely to be invalid, in which case it cannot be shown that any one of the two models is more appropriate to describe the haemoglobin-oxygen system.

In another article, Perutz and Shibata and their co-workers have re-investigated the Japanese haemoglobin variant Hb-Hiroshima, in which the magnitude of the Bohr effect is halved, the oxygen affinity at physiological $p \mathrm{H}$ is increased three times and the haem-haem interaction is somewhat reduced. This variant was thought to have the substitution $\beta 143$ (histidine $\rightarrow$ aspartic acid), but the normal effect of 2,3-DPG on the oxygen affinity of $\mathrm{Hb}$-Hiroshima was inconsistent with the proposed role of this residue in the binding of 2,3-DPG by normal adult haemoglobin.

Perutz and his colleagues have now been able to show, both by X-ray crystallography of deoxy Hb-Hiroshima and by chemical methods, that the replacement is in position $\beta 146$. The physiological properties of $\mathrm{Hb}$-Hiroshima can now be accounted for without difficulty. The result also confirms the earlier suggestion that half the alkaline Bohr effect is due to the salt bridge between the imidazole side chain of $\beta 146$ (His) and the $\gamma$-carboxyl group of $\beta 94$ (Asp). The reduced haem-haem interaction in Hb-Hiroshima is also related to the absence of a histidine residue at $\beta 146$, which affects both the tertiary structure of the $\beta$-chains and the quaternary structure of the entire molecule. 